\title{
Behavioral Intention to Use IoT Health Device: The Role of Perceived Usefulness, Facilitated Appropriation, Big Five Personality Traits, and Cultural Value Orientations
}

Tommy Prayoga, Juneman Abraham

Department of Psychology, Bina Nusantara University, Indonesia

\section{Article Info}

Article history:

Received Mar 20, 2016

Revised May 18, 2016

Accepted May 30, 2016

\section{Keyword:}

Appropriation Internet of things Personality and culture Psychotechnology Technology acceptance

\begin{abstract}
As one of the biggest technology breakthroughs, Internet of Things (IoT) has been gaining attentions from industries. Especially in the healthcare sector, IoT health devices have a great impact on individual's health-regulation. Through many beneficial features from tracking sleep patterns to calories intake measure, individuals will gain a better understanding of their body to keep them healthy. However, benefits do not immediately equal users' acceptance. In this study, we seek to find variables that we propose could predict user's intention to use IoT health device and integrate them into a theoretical model. We analyzed user's technology acceptance from the perspective of Technology Acceptance Model, using Perceived Usefulness as the main predictor for Behavioral Intention. We also integrate Personality Traits and Facilitated Appropriation as factors to determine Perceived Usefulness, and finally using Cultural Value Orientations at the individual level, we seek to determine the antecedents of Facilitated Appropriation. The research design was predictive correlational with path analysis statistical technique. Participants of this study were 186 college students ( 75 males, 111 females; mean $=20.86$ years old, standard deviation $=2.01$ years) across Greater Jakarta. The results showed that Perceived Usefulness can predict Behavioral Intention to use the device. However, Personality Traits are found to be irrelevant in predicting Perceived Usefulness. Only Facilitated Appropriation predicts Perceived Usefulness. Among cultural values proposed, only the Long-term Orientation predicts user's tendency to engage in Facilitated Appropriation. Also, we did not find any correlation between age and Behavioral Intention, Perceived Usefulness, and Facilitated Appropriation.
\end{abstract}

Copyright (C) 2016 Institute of Advanced Engineering and Science. All rights reserved.

\section{Corresponding Author:}

Juneman Abraham,

Department of Psychology,

Bina Nusantara University,

Jl. Kemanggisan Ilir III no. 45, Palmerah, DKI Jakarta 11480, Indonesia.

Email: juneman@binus.ac.id

\section{INTRODUCTION}

We are no stranger to the phrase, "Technology makes our life better-or easier, or more efficient." It is the situation as we seek new ways to improve our life with daily technological advances, inventions and innovations. The idea of a system that let us attain higher objective in life with less and less effort has become inseparable in modern human society. Although the idea is simple, the phrase itself contains almost infinite possibilities of what we can do with technologies just by simply questioning the technology in use, which part of our life it could improve, and the extent it makes life better or efficient. This argument of possibilities was based on the ground-breaking idea of the Interconnected Network of Things or IoT technologies. It is a concept reflecting a connected set of anyone, anything, anytime, anyplace any services 
and any network [1]. The idea was to give any object the capabilities of identifying, sensing, networking and processing with other objects over a network to accomplish some purposes [2].

Currently, IoT is thoroughly applied and being developed in every area of life and industry. One of the most important areas that we will discuss in this article is the application of IoT in the Healthcare systems. In healthcare systems, IoT is mainly built to access and analyze the large scale of health information by taking advantage of its high potential features of flexibility, adaptability, high speed and cost shrinkage [3]. For example, the currently operating cooperative medical diagnosis multi-agent system called CMDS (Contract Net Based Medical Diagnosis System) can solve flexibly a large variety of medical diagnosis problems [4] because it has more autonomy for human and artificial intelligence agents to cooperate and solve problems [5].

IoT devices do not only benefit health institutions and medical patient but also to the healthy individual. Health trackers, for example in the form of built-in apps, armband and chips are the examples of IoT devices commonly used to help individuals in self-regulating their health. To self-regulate means to plan, control and carry out conscious behavior consistently to accomplish a goal [6]. In the context of health behavior, it may take forms such as having a diet to reach certain sugar levels, body shape or body weight. It relies greatly on the ability of individual to maintain the behavior on an on-going basis, their appropriation of knowledge to execute the task and to engage in health goal-directed behavior [7]. All of these processes can be enhanced and facilitated by IoT devices.

The role IoT health devices may play is providing body health information, goal suggestion, tracking diet progress, timeline milestones by presenting clearer criteria and positive outcomes for them to hold on to reach the goal. For example, if your body experiences any unusual changes such as faster heartbeat, rise in blood sugar or blood pressure, the device will track it and record it, and send signals to your phone to notify you suggesting safety actions you could engage in, informing your doctors, your family and friends [8]. Here, by IoT devices, knowing your body conditions is subsequently followed by engaging in a health negotiation as the device acts as a consulting or negotiating party with alternatives provided to the user. Users have the freedom to choose the alternatives or even ask for other alternatives (instead of engaging in steps provided). The negotiation process involves psychological processes (perception, cognition, power, influence, emotions, communications, and ethics) of the users, which influence strategy used by them to deal and reason with (1) the information and options provided by the device, and (2) the outcome of that negotiation [9], which eventually impacts their decision on their self-regulation.

Imagine if you could keep track of the chemical components in your blood, change how you sleep every day or night and gain more personal understanding of your body. Imagine you know how much weight you are gaining or losing, the number of calories you consume and burn, and finally, know how much you are progressing towards your health goals. These are only a few examples of the impact IoT health devices would have in our lives. It can change individual's construal about current immediate behavior consequences to be more consistent and less distant with their more abstract and further ultimate health goal (compare also with [7]), letting people know how they are progressing and how significant it is.

As promising as it sounds, implementing IoT in healthcare sector still needs to overcome the issue of acceptance by intended users. We are not talking about the devices and systems that are found in medical institutions, but rather the beneficial IoT health devices that are more optional (people can choose to or not to have it) such as health trackers. A device or system may possess tremendous benefits for people who seek better health, but the lack of acceptance may hinder its purpose. In endorsing new technologies to the users, considering factors that influences acceptance would help to promote the use of IoT healthcare devices.

The best way to look at that issue is from the perspective of Technology Acceptance Model or TAM [10]. TAM has been the most widely accepted model in explaining psychological and environmental factors that affect individual's technology acceptance and adoption [11]. According to TAM, the outcome component that indicates acceptance is Behavioral Intention (BI), which signifies individual's intention to use the technology before exhibiting actual using behavior [12]. Simply put, if individual's BI is higher, the tendency to use the technology is also higher.

As professionals who understand the benefit IoT devices have on health, the hope is of course to maximize behavioral intention to use the device. However, as there is great diversity in reactions to many different technologies with different functions [13], introducing new technologies to fit people's need and be accepted is very challenging. The diverse reactions may come from the difference in individual's personality, environment, culture values and other factors. By taking into account and understanding these factors, we can base further actions in improving technologies and the acceptance of these factors [14].

The purpose of this study is to outline some of the considered important predictors of individual's behavioral intention to use IoT health devices into a theoretical model. We gathered adolescents (12-18-yearold) and young adults (18-35-year-old) attending college across well-known-IT (information technology)campuses in Greater Jakarta to participate in the study. The reason to sample this demographic are: teenagers 
and young adults in college are the largest user of internet and smart gadgets, and they also play a role in the trend and virality of many latest technologies [15]. Second, as there are no age restrictions in enrollment, even the students in bachelor's program have a large age variety. Any individuals with decent high school diploma that fits into the category of teenagers and young adults can enroll in the IT study program if they pass the selection process.

We will start by discussing the Behavioral Intention in Technology Acceptance Model. As one of the most prominent model that has been tested, applied, refined and validated many times in explaining the acceptance of different technologies [16], the key component of TAM is the behavioral intention (BI) to use technology.

Among many factors that influence BI in the model, perceived usefulness (PU) has been viewed as the main predictor of BI. People who intend to use a particular technology end up using this technology to a larger extent (than those who do not), and those people are who find a technology useful [17]. Perceived usefulness can be defined as the extent to which a person believes that using a particular system would be helpful and enhance performance [10]. In the context of IoT health devices, users that perceive a device as useful means see that it benefits their health and thus helping them achieve their health goals, resulting in more likely acceptance and use. In this study, we propose PU as the primary antecedent that predicts BI. Thus, we form our first hypothesis, "One's Perceived Usefulness towards IoT health Device can predict his/her Behavioral Intention to use the device" (H1).

Next, we seek to determine what influences Perceived Usefulness. Individual's personal characteristics or traits could influence their perception of technology usefulness [18]. We suggest that Perceived Usefulness is subjective and personal, and we propose that, in the context of accepting new technology, it could be determined by basic individual differences, that is personality.

In recent years, many information system studies have incorporated personality traits in attempts to explain user's technology acceptance [14] which has also been demonstrated by numerous studies (e.g. [13][14],[19]). The reason to examine personality traits in the study of technology acceptance is because the basic concept underlying the model places significant focus on individual's reactions [20] in which personality traits is expected to play an important role in influencing the decision to accept the technology. The Theory of Reasoned Action as the basis for technology acceptance model also explicitly incorporated personality traits as an external variable affecting an individual's belief towards technology [20]-[21]. Based on those propositions, it's logical to assume that the role of personality here is influencing individual's belief of technology's usefulness. Moreover, as the potential users of technology may vary greatly, differentiating personality regarding technology use and acceptance is important to do [22].

From the perspective of traits, to our knowledge, the best conceptualization of personality is the Five-Factor Model of personality [23], also called the "Big Five Model." Goldberg designed it in 1982, which later became the basis of proposing that individual's characteristics can be described regarding their scores on five personality domains including openness, conscientiousness, agreeableness, extraversion and neuroticism [24]. Some researches that study the impact of personality traits on technology acceptance were compiled [19], and among those studies, there was a various degree of prediction from each variable towards perceived usefulness of many different kinds of technologies. Thus, in this study, the authors are also going to examine the ability of all five variables in influencing perceived usefulness of IoT health devices.

We will first discuss the Openness trait. The openness trait delineates the tendency to be imaginative, independent, and interested in variety rather that practical, conforming routine [25], and it characterizes an individual's approach to new situations [17]. Interest in novelty fosters the willingness to build up in intention to use new technology, making people with dominant openness trait tend to welcome novel situations or object better [13]. For example, when they are introduced with new technologies that provide new ways to regulate their health, they will be less likely to hesitate and more likely to try them than less open individuals. We argue that as 'open' individual is more curious and welcoming, they are also more receptive to new technology, even so when the technology is beneficial to something as pivotal and personal as health. Thus, we hypothesize, individual with higher Openness trait is more likely to perceive IoT health device to be useful. In other words, "One's Openness trait can predict his/her Perceived Usefulness towards IoT health device" (H2).

The second trait we are going to discuss is Agreeableness. It can be seen as the tendency to be helpful and to trust [25]. Agreeableness is associated with compliance, modesty, and tender-mindedness [26]. They are more likely to be accommodating and cooperative positively when considering a new technology [20]. For example, to achieve their goals and attain the benefits that the technology provides, they will eagerly try to understand the features, be prepared to utilize it, work with the manuals and instructions, and use the technology accordingly. Devaraj, Easley and Crant [20] indeed found a direct effect of Agreeableness on Perceived Usefulness. Punnoose [19] also found that agreeableness has a significant positive direct effect on PU. We argue that the tendency to cooperate and to accommodate would make them welcome helpful 
technology better. This is due to the reason that IoT device, especially health tracker, are not merely a tagged device, but rather a negotiator party to make decisions and achieve goals. If they can work with-meaning if technology can be applied in a way that aligns with their characteristic - they will perceive the technology as useful. Thus, we argue in this study, individual with higher agreeableness trait will be more likely to perceive IoT health devices as useful because the beneficial nature of the devices towards their health pair well with the accommodating and cooperative nature of agreeable individuals. In other words, "One's Agreeableness trait can predict his/her Perceived Usefulness towards IoT health device" (H3).

The third trait we propose could predict perceived usefulness is Extraversion. The trait is associated with warmth, gregariousness, assertiveness and excitement seeking [27]. In the context of technology acceptance, extraversion has been associated positively with PU [17],[22],[24]. There is a lot of energy and readiness in the trait of extraverted that will overcome doubts about using technology [13]. Extraverted individuals will be more likely to perceive technology as useful if they could assert themselves through it [22]. We argue it is also possible for an individual to assert themselves through an IoT health device, as it doesn't only serve personal health information that might be previously unknown to the users (such as blood pressure, lung capacity and sleep patterns), but it also allows users to interact socially with other users in sharing data, competing and achieving a goal together. Thus, we propose that extraverted individuals will also perceive IoT health device as useful. In other words, "One's Extraversion trait can predict his/her Perceived Usefulness towards IoT health device" (H4).

The next trait we are going to discuss is Conscientiousness. Conscientiousness is a trait associated with self-discipline, deliberation, dutifulness, order, competence, and achievement striving [23]. Conscientious individuals are intrinsically motivated to achieve life's objectives, perform at high level and take actions to improve their performance whenever possible. Those characteristics could also be applied to maintain health. Conscientiousness individuals are rule followers, persistent, thus are better at following directions and find proposed technology is easier to be accepted [22], due to their nature to follow concrete, clear instructions. This trait has a strong influence on perceived usefulness of technology [20],[24]. We suggest this finding is due to the natural tendency of a conscientious individual to maintain order and struggle for goals, thus, if technology can be applied in a way that aligns with their characteristics, it will be perceived as useful. IoT health device, along with its abundant features and benefit, provides information and instructions on how to attain them. With those instructions that are clear and concrete enough to be understood practically, conscientious individuals will find the device more useful. Moreover, our arguments were supported by Svendsen et al. [17] stating that Conscientiousness can influence Behavioral Intention, but only through the mediation of belief, such as Perceived Usefulness, especially in a "more personal" application context, such as in IoT health device. Thus, we argue in this study, individual with higher conscientiousness trait will perceive IoT health devices as more useful. In other words, "One's Conscientiousness trait can predict his/her Perceived Usefulness towards IoT health device" (H5).

The final trait we are going to discuss is Neuroticism. It is commonly linked with anxiety and insecurity, and individual with dominant neuroticism trait is more likely to have less self-esteem and selfefficacy [22]. When faced with "relatively new technologies" they are unfamiliar with, like IoT, neurotic individuals are more likely not to use it to avoid uncertain outcome or risk [19], such as failing to understand the device's purpose, wasting unnecessary time and energy, or even the devices ended up affecting or changing their lives in a negative way. Moreover, neurotic individuals are less likely to perceive a device to be useful [20]. Punnoose [19] argued that this is due to the generalization from their negative view towards situations in life. Neurotic individuals will seek stable and more fixed information to comfort their insecurity. Although the technology they are unfamiliar with offers great benefits, if it requires doing certain new efforts, they will be less likely to perceive it as useful. In other words, "One's Neuroticism trait can predict his/her Perceived Usefulness towards IoT health device" (H6).

Aside from personality traits, another important element that we want to integrate into this study is the concept of appropriation and facilitation in influencing perceived usefulness. Appropriation, as an important sign of technology acceptance, can be defined as the improvisation in using technology in ways it was not designed to be, to fit the user's need [27]. On the other hand, Technology Facilitation can be defined as the facilitating conditions that support the likelihood of technology being use or appropriated [28], like technology, networks, and peers' help.

Unlike other studies on already popular applications, networks, and devices that differentiate facilitation and appropriation in explaining technology acceptance, in personal IoT health devices such as mentioned in the previous section, we view the way technology will be appropriated by its user is inseparable from the facilitation of the device. This is based on the argument by Bostrom, Anson, and Clawson [29] stating that the technology itself will provide facilitation capabilities, and users, through the technology, could take advantage of this capability. The simplest form of facilitation could be a guide for the features, not to mention IoT devices, also serves the purpose as a negotiator in helping the user to make a decision on what 
to do with the device and their health. Rather than seeing facilitations as antecedents to appropriation, we propose that it is a part of the appropriation process. Here, we use the term "Facilitated Appropriation" to describe this process better. Facilitated Appropriation can be seen as a more comprehensive and systematic appropriation. The appropriation is influenced by factors that facilitate, namely information, guidelines and other ways of appropriation. Therefore, more variety and versatile alternatives of appropriating in meeting goal's end occurs as user's creativity is heightened by the facilitation.

Understanding technology acceptance as appropriation means recognizing that a user is an active agent who can adapt technology to serve personal or shared goals when needed [30]. With the right amount of information and supports (facilitations), appropriation has the potential to give additional values and benefits to the devices asides from how it is designed. This is because while engaging in facilitated appropriation, users are inspired to see and have additional features on the device, exclusively dealing with their problems or other personal purposes. For example, using sleep pattern tracker not only to attain better sleep, but also to plan schedules and activities ahead, and even to allocate the energy spent to focus on tasks users wish to accomplish. We propose that if users feel they are facilitated to appropriate IoT health device, they would be more likely to view the technology as useful. In other words, "One's Facilitated Appropriation can predict his/her Perceived Usefulness towards IoT health device” (H7).

The final element we feel essential to be included in our model to explain user's acceptance is Cultural Orientations. While in the previous sections we argued that individual's belief of the technologies perceived usefulness is influenced by innate factors, such as trait dispositions, beliefs and tendency to act upon it by their own way (to appropriate), here we propose those innate personal factors, in turn, are influenced by macro factors, that is the culture. All those factors that were dependent on social context and environment are the manifestations of culture [31]. Here, we argue that culture impacts what kind of information an individual get, value, and hold onto when using the technology, which will be very likely to influence how he/she appropriate the technology. To explain the role that culture plays in this context, we need to use the lens of the Hofstede's Cultural Dimension that differentiates cultures regarding a few dimensions, such as collectivism, power distance, masculinity degree, long-term orientation and tendency to avoid uncertainty [32] in individual level.

The first factor of cultural dimension we thought could influence technology appropriation is collectivism. Individuals in collectivistic culture are inter-dependent, meaning that activities such as work, sharing information and introducing objects are done together between social circles and at all community levels [33]. Collective individuals value member participation, social relationship, and striving towards a shared goal [34] in daily activities or when faced with new changes. Due to their characteristics, we argue that collective individuals will be more likely to share and discuss information about technology, hence triggering and reinforcing the facilitated appropriation. For example, upon finding a new way to use certain features on the device, they will be more likely to tell their peers and cohorts, or upon facing problems, finding solutions together through appropriating the device. The problems will be likely to converse about among collective individuals, e.g. considering the pros and cons of the IoT device. On the other hand, individuals with lower collectivity, or individualistic individuals, will be less likely to share information [35] among members, making them less be driven to try out or appropriate the technology. In other words, "One's degree of Collectivism can predict his/her Facilitated Appropriation tendency towards IoT health device" (H8).

Aside from collectivism, we propose that Power Distance - the power gap between authority/person in charge and subordinates/people in society-might influence how individual appropriate technology. It describes how much influence one party in the different hierarchy has towards another in determining their behavior in all aspect of social life [36]. The higher gap means more unequal power distribution, and lower hierarchy society member should comply with higher-ups. Power distance is also associated with the degree of autonomy individuals have in maintaining society's order [37]. Although not necessarily limited to the higher-ups, we argue that individual that justifies a higher power distance will be less likely to appropriate technology, as they will be more susceptible to instructions or example provided. For example, in using new technology, they will be more likely to use it as how the higher-ups in place use it, or instruct them on how to use it, as less autonomic thought and act could be expected. In other words, "One's degree of Power Distance can predict his/her Facilitated Appropriation tendency towards IoT health device" (H9).

The next factor that we propose could predict user's appropriation is the masculinity. This dimension or orientation does not refer to the male or female dominance in a society nor explains any genderrelated differences [31], rather it depicts the degree to which masculine traits like authority, assertiveness, performance, and success are preferred to feminine characteristics like personal relationships, quality of life, service and welfare [35]. Individuals who espouse masculine values emphasize work goals such as earnings, advancement, competitiveness, performance, and assertiveness [31]. We argue that these characteristics are manifested in their behavior, including on how individual will appropriate technology. Individuals that 
prioritize advancement and performance are expected to maximize the function in a technology and will be more likely to search for ways to appropriate it so that they will reach their goal or perform better. On the other hand, individuals with lower masculinity degree are associated with voluntary status-accord to those who refrain from using or showing power, and a reluctance to use power, or to accept authority when it is enacted in powerful ways [38]. We argue individuals with lower masculinity traits will be less likely to use their 'power' to maximize the use of IoT devices. They are more likely to follow what are told on how to use the device by the original instruction; therefore, they will be less likely to appropriate. In other words, "One's degree of Masculinity can predict his/her Facilitated Appropriation tendency towards IoT health device" (H10).

The next dimension we propose to be able to predict individual's Appropriation is the dimension of Uncertainty Avoidance. It is a concept describing individual's attitude towards risk and uncertain stimuli, whether people feel threatened and hence try to avoid it [39]. Individual with higher tendency to avoid uncertainty will require more structures and guideline in a situation to reduce their uncertainty [35]. They would prefer stable situations, traditional way, and are less likely to seek a new way to deal with things. An individual with lower uncertainty avoidance tendency will be comfortable in novel situations, such as new technology and will be more likely to use technology in new ways; for example, try to use blood pressure or blood sugar tracker to test their limit of sugar intake (or outtake) or even their exercise limit, which is potentially dangerous. Thus, we argue that individual's tendency to avoid the uncertain can predict their tendency to appropriate technology. In other words, "One's degree of Uncertainty Avoidance can predict his/her Facilitated Appropriation tendency towards IoT health device” (H11).

The final cultural dimension we propose is the Long-Term Orientation. It can be seen as valuing prospect of the future, and deeming actions unimportant for the short-term achievement [40]. Individuals in a society that value long-term orientation does not only see the future, but also the concomitant influence of present and the past [41]. They prepare for the future from now, and current effort and resources are invested in something that will be obtained ahead, such as their health goals. We argue that this characteristic also applies to achieving their health goal using IoT device, as an individual long-term oriented individual would be able to see the future benefit and be more committed. Health goals are usually achieved by long term and periodical effort, such as losing weight, gaining mass, sleep better and lowering blood sugar intake, which is unattainable with short-term orientation planning. These goals certainly align with individuals with a longterm orientation. For them, the clearer the future goal is, the more they will invest their energy to achieve it, e.g. through facilitated appropriation. We propose that long-term oriented individuals will be more likely to maximize the appropriation of any given IoT health device to fit their goal. In other words, "One's degree of Long-Term Orientation can predict his/her Facilitated Appropriation tendency towards IoT health device" (H12).

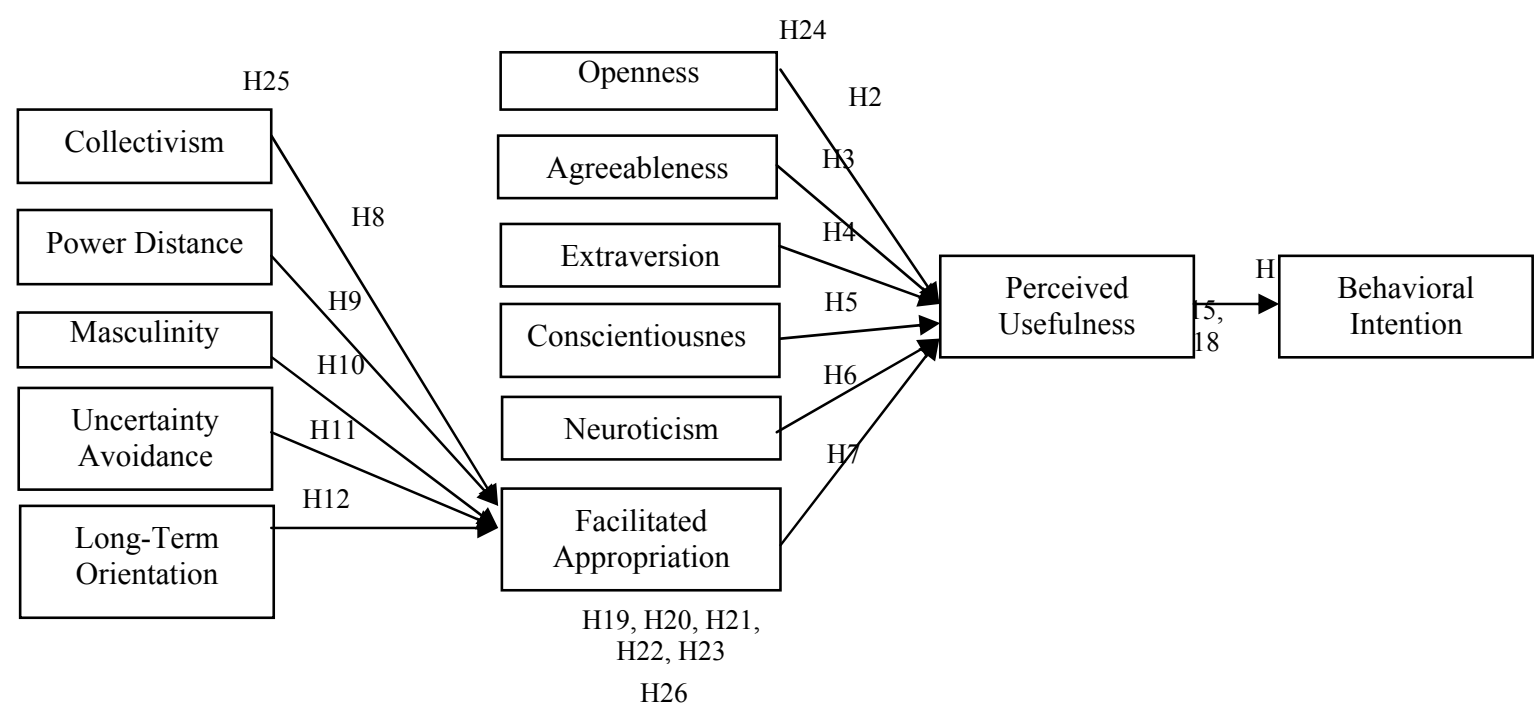

Figure 1. Hypothetical Model 
On the model above, arrows indicate prediction made on each hypothesis. Also, as we wanted this model to explain plausibly the Behavioral Intention, there are a few hypotheses we would like to test. First, Perceived Usefulness as one of the in-between variables in this study should be able to mediate the relationship between each personality traits and Facilitated Appropriation, meaning that in order to to use an IoT health Device, individual with aforementioned traits and appropriation tendency must believe that the device is useful first, then they will use the technology. If they don't feel it as useful, they will not have the intention to use it. The hypotheses formed are as follow.

H13: "One's Openness traits can influence his/her Behavioral Intention but only through the mediation of Perceived Usefulness."

H14: "One's Agreeableness traits can influence his/her Behavioral Intention but only through the mediation of Perceived Usefulness."

H15: "One's Extraversion traits can influence his/her Behavioral Intention but only through the mediation of Perceived Usefulness."

H16: "One's Conscientiousness traits can influence his/her Behavioral Intention but only through the mediation of Perceived Usefulness."

H17: "One's Neuroticism traits can influence his/her Behavioral Intention but only through the mediation of Perceived Usefulness

H18: "One's tendency to engage in Facilitated Appropriation can influence his/her Behavioral Intention but only through the mediation of Perceived Usefulness."

Second, we also propose cultural value orientations in the individual level could have an influence on perceived usefulness and eventually have an impact on their behavioral intention. However, the effect culture has on perceived usefulness must be mediated by the tendency to appropriate. We argue that the perceived technology usefulness is obtained through the perception of successfully facilitated appropriation, which is strongly affected by cultural values. So, to see culture's effect on individual's belief, an individual must have the tendency to appropriate the technology first. Thus, we form additional hypotheses:

H19: “One's degree of Collectivism can influence his/her Perceived Usefulness but only through the mediation of Facilitated Appropriation."

H20: "One's degree of Power Distance can influence his/her Perceived Usefulness but only through the mediation of Facilitated Appropriation."

H21: "One's degree of Masculinity can influence his/her Perceived Usefulness but only through the mediation of Facilitated Appropriation."

H22: "One's degree of Uncertainty Avoidance can influence his/her Perceived Usefulness but only through the mediation of Facilitated Appropriation."

H23: "One's degree of Long-term Orientation can influence his/her Perceived Usefulness but only through the mediation of Facilitated Appropriation."

For the model to be plausible, we need to confirm that every acting predictor in the model proposed can predict the criterion variables significantly.

H24: "All Personality traits and Facilitated Appropriation can simultaneously predict Perceived Usefulness."

H25: "All cultural orientation dimensions can simultaneously predict Facilitated Appropriation."

Last but not least, based on the variables integrated into this study, we propose a theoretical model to explain user's Behavioral Intention in using IoT health Device. Based on the arguments made each hypothesis, Figure 1 is the visual representation of the model, with arrows indicating predictions.

H26: “All variables used in this study can be used to explain user's Behavioral Intention in using IoT health device by the theoretical model proposed."

\section{RESEARCH METHOD}

\subsection{Participants and design}

The sample for this study was 186 college student of many different faculties and/or study programs from well-known-IT campuses (most of them came from Bina Nusantara University, Jakarta, and Multimedia Nusantara University, Tangerang, in Indonesia), such as humanities, art, computer science, engineer, 
psychology, and business management. There were 75 male participants and 111 female participants (Mean of age $=20.86$ years old; Standard deviation of age $=2.01$ years; Range of age $=12-30$ years old) recruited through convenience sampling technique. Almost all of them are smartphone users, and have accounts in various social networking sites, yet only a little portion of $11 \%$ heard of IoT health tracker device.

The design of this study was predictive correlational with path analysis statistical technique, as we seek to analyze the prediction relationship between each hypothesized variables in the model. Here, we use the psychological scales in a questionnaire to gather participant's respond. However, this study did not ask participants' responds as a user in the actual use of technology, but rather through reviewing one of the latest IoT health device described. The main reason for using a description of the hypothetical situation instead of employing an actual technology was to obtain a large and representative sample without incurring prohibitive costs [17]. After participants had comprehended the features of IoT device, they were asked to fill in their response to the questionnaire. The detail of the text and how it is used will be detailed in the next section.

\subsection{Materials and Procedure}

We used the criteria of Cronbach's Alpha $\geq 0.600$ as the index of internal consistency to determine the reliability of each psychological scale, and index of corrected item-total correlations $\geq 0.250$ to determine the item validities. To test the reliability and validity of the measurement instrument, this study recruited 75 students across faculties for instruments try-out phase. The questionnaires given to participant were all translated into Indonesian and adapted to fit the health context; all questionnaires passed the reliability (each presented in parentheses) and item validity test.

Behavioral Intention and Perceived Usefulness constructs were measured using the adaptation of a model used in the study of consumer acceptance of IoT by Gao and Bai [42]. We adapted and reworked the items on the subscales to fit the context of this study. First, we added a narration (in Indonesian language) describing the features and utility of a new health tracker device, UP3 by the company Jawbone, as follow.

\section{UP3 by JAWBONE}

One of the latest technology breakthroughs in the health sector is UP3 health sensor by Jawbone (see Figure 2). It's a wristband with a screen that contains many health-supporting features. Using Bluetooth, we can synchronize it with other smart devices and applications to provide the user with tremendous health benefits. First, it has the ability of Activity Tracking that track and provide information about our activities, such as steps taken, exercise done and calories burned. Advanced Sleep is for tracking sleeping stage and cycle, to optimize sleep pattern. Smart Alarm informs about the most suitable sleeping time and wakes user in the right time to provide freshest waking experience.

Another unique feature is Food Logging that measures calories intake, scanning restaurant barcode that shows rating in calories and nutrition to help user form a better eating pattern. Smart Coach is good for motivating and directing the user to achieve the goal. Also, the longer we use the device, the more it understands our pattern and lifestyle and will fit updates and suggestions according to our records. The feature Today I Will challenges user to do certain beneficial activities, such as consume vitamins and drink more water. Heart Health is used to track primary organ function like heart and liver; Idle Alert is to remind user if they stay inactive, like sitting for too long.

UP3 does not only benefit health, but it can be seen as a social device as information and progress can be shared from users to users, challenging each other in achieving goals and get rewards using the Duel feature. UP3 works by comparing individuals in the same category in real time so that the feature works accurately. Other technical features in this 29 gram band are: the 168 hours lasting battery without charging, waterproof, automated Bluetooth upload, record data sharing, saving and even removing, milestone \& streaks that estimate distance towards a goal, and Finally it can connect to user's social media as posting, tweeting and another method of sharing information is available.

(adapted from: http://www.ebay.it/itm/Jawbone-UP3-Stylish-Fitness-Tracker-Bluetooth-Sync-Health-Sleep-Activity$/ 171845748006)$

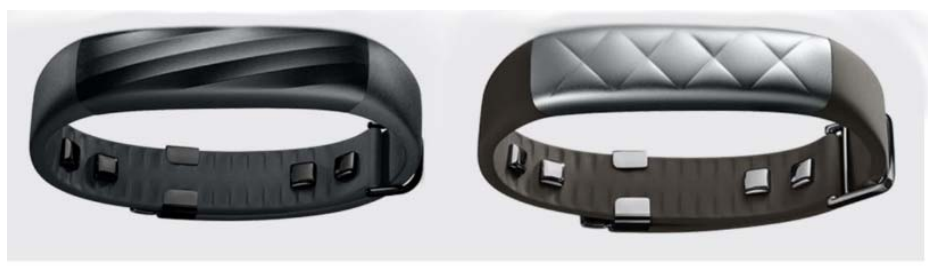

Figure 2. Jawbone UP3

(source: https://www.youtube.com/watch?v=lLgdD31AEhI) 
We then asked the participant to five response to 4 item statements for BI $(\alpha=0.891)$ and 6 item statements for PU $(\alpha=0.947)$ that is related to the text. Examples of the items were: "I will use UP3 product" and "I'm willing to use UP3 product" for BI; and "Overall, I feel the UP3 product is beneficial to me" and "Using UP3 product will improve my health quality significantly" for PU. The participant was asked to indicate their response in agreement toward each statement by choosing the one of the seven verbal response Likert scales from "Strongly disagree" (score of 1) to "Strongly agree" (score of 6). None of the items were omitted from the validation process. The range of corrected item-total correlations is 0.689 to 0.904 .

Personality traits were measured using The Big Five Inventory (BFI-44) [43]. The questionnaire originally contained 44 items in total, but we only selected items from each trait: Agreeableness (9 items), Openness (10 items), Conscientiousness ( 9 items), Extraversion ( 8 items) and Neuroticism ( 8 items). Through validation process, 5 items were omitted from Conscientiousness scale $(\alpha=0.850), 4$ items from Extraversion $(\alpha=0.792), 2$ items from Openness Scale $(\alpha=0.841), 6$ from the Agreeableness scale $(\alpha=$ $0.760)$, and 4 from the Neuroticism scale $(\alpha=0.729)$, resulting 23 items in total. Participant were asked to indicate a score from 1 ("Do not describe me at all") to 6 ("Describe me very well") towards every statement or phrase regarding themselves, such as "I am a quiet person" describing Extraversion, "I have an active imagination" describing Openness; "I'm unorganized" and "I'm lazy" (unfavorable items; reversedly scored) describing Conscientiousness; "I'm emotionally stable and not easily angered" (unfavorable items; reversedly scored) describing Neuroticism, and "I'm forgiving" describing Agreeableness. The range of corrected item-total correlations for each personality subscales is 0.299 to 0.839 .

Facilitated Appropriation were measured using the adaptation of facilitation and appropriation measure of the study of Lin [28]. Like the previous instrument, we also adapted and reworded the items to fit the study context and used the same description of the same product. Adapted were six items measuring facilitated appropriation $(\alpha=0.873)$. Examples of the items were "I understand the information given by the product to help me with my health goals" and "I will use the product to regulate my sleep pattern." The participant was asked to indicate their response in agreement toward each statement by choosing the one of the seven verbal response Likert scales from "Strongly disagree" (score of 1) to "Strongly agree" (score of 6 ). None of the items were omitted from the validation process. The range of corrected item-total correlations is 0.580 to 0.780 .

Finally, we measured cultural value orientations (in individual level) from using the combination of subscales from Personal Cultural Orientations instruments [44] and Cultural Value Scale or CVSCALE [45]. Masculinity was measured using Personal Cultural Orientations only. There were 15 items measuring Power Distance, 15 items measuring Uncertainty Avoidance, 14 items for Collectivism, 10 items for Masculinity and 8 items for Long-Term Orientation. Through validation process, 5 items were omitted from Collectivism subscale $(\alpha=0.791), 6$ from Power Distance subscale $(\alpha=0.826), 1$ from the Masculinity subscale $(\alpha=$ 0.729), while no items were omitted in Long-Term Orientation subscale $(\alpha=0.764)$ and Uncertainty Avoidance subscale $(\alpha=0.861)$, resulting in 50 items in total. Example of the items were "I feel stressed out if I can not predict the outcome of a situation" for Uncertainty Avoidance, "The well-being of a group is more important than individual's well-being" for Collectivism, "Long-term and careful planning is my priority" for Long-term Orientation, "Person in higher position should not ask a person with lower position for advice" for Power Distance, and "Men are more logical in solving problem, and women are more intuitive" for Masculinity. The range of corrected item-total correlations is 0.307 to 0.692 .

\section{RESULTS AND ANALYSIS}

The demographic descriptions of the participants of this study are as follow: there were 75 male and 111 female participants, with $44 \%$ of them 16 to 20 -years-old, $54 \%$ of them 21 to 24 -years-old and $2 \%$ of them 25 to 30 -years-old. All 186 participants possessed gadgets, such as smartphones, and similar devices. Regarding health tracker, $91 \%$ thought it is helpful for achieving personal health goals, $89 \%$ believed it could change people's health pattern, and $90 \%$ thought it will revolutionize the healthcare systems. Although $87 \%$ of them had searched information about health-related information online and $35 \%$ had heard about health trackers technology, only $13 \%$ had actually used it.

Assumption test for path analysis result showed that the data is free from heteroscedasticity, all independent variables are free from multicollinearity, and the data distribution is normal. For Path Analysis, we looked at each prediction's path coefficient magnitude and $t$-value significance using LISREL 8.8. For $\alpha$ at the 0.05 level, we used $t=|1.96|$ as a critical value for judging statistical significance of the path coefficients [46] (see Figure 3). We will present each value in a format of: (coefficient estimation, $t$-value).

RMSEA indicates a value of $0.016(R M S E A<0.05)$, with $P$-value $0.400(P$-value $>0.05)$ of $\chi^{2}=$ 21.99; all indicates this model has a good fit and can be used to explain Behavioral Intention. H26 was supported by empirical data.

Behavioral Intention to Use IoT Health Device: The Role of Perceived Usefulness .... (Tommy Prayoga) 
In this study, we found that Perceived Usefulness can predict Behavioral Intention in a postive way $(\beta=0.68, t=15.68, t>1.96)$. H1 was supported by empirical data. For Perceived Usefulness, we only found significant positive prediction from Facilitated Appropriation $(\beta=0.60, t=16.30, t>1.96)$; while Extraversion $(\gamma=-0.02, t=-0.38, t<-1.96)$, Agreeableness $(\gamma=0.02, t=0.30, t<1.96)$, Conscientioussness $(\gamma=0.05, t=0.88, t<1.96)$, Neuroticism $(\gamma=-0.02,-0.33, t<-1.96)$, and Openness $(\gamma=-0.03, t=-0.84, t<$ -1.96) can not predict PU. H7 was supported by empirical data. H2-H6 were not supported by the data. The effect sizes are shown in Equation 1 below.

There are no significant predictions of Facilitated Appropriation by Uncertainty Avoidance $(\gamma=$ $0.00, t=0.00, t<1.96)$, Power Distance $(\gamma=0.04, t=0.77, t<1.96)$, Collectivism $(\gamma=0.08, t=1.03, t<$ 1.96) and Masculinity $(\gamma=0.05, t=0.82, t<1.96)$. Facilitated Appropriation is only predicted by LongTerm Orientation in a positive way $(\gamma=0.24, t=2.66, t>1.96)$. H12 was supported by empirical data. H8-H11 were not supported by the data.

As the corollary of the above findings, it is known that Facilitated Appropriation and all Personality traits can not simultaneously predict Perceived Usefulness, and all Cultural Orientations can not simultaneously predict Facilitated Appropriation. H24-H25 were not supported by empirical data.

We also found that only Facilitated Approrpriation has an indirect effect on BI (Total effect $=0.40, t$ $=11.30, t>1.96)$; while Extraversion (Total $=-0.01, t=-0.38, t<-1.96)$, Agreeableness $($ Total $=0.02, t=$ $0.30, t<1.96)$, Conscientioussness (Total $=0.05, t=0.88, t<1.96)$, Neuroticism $($ Total $=-0.01, t=-0.33, t$ $<-1.96)$, and Openness (Total $=-0.02, t=-0.84, t<-1.96)$ do not. Only Long-Term Orientation $($ Total $=$ $0.10, t=2.59, t>1.96$ ) has an indirect effect on Perceived Usefullness; while Uncertainty Avoidance (Total $=0.00, t=0.00, t<1.96)$, Power Distance $($ Total $=0.02, t=0.77, t<1.96)$, Collectivism $($ Total $=0.05, t=$ 1.03, $t<1.96$ ), and Masculinity (Total $=0.03, t=0.82, t<1.96)$ do not. This means that Perceived Usefullness only mediates Facilitated Appropriation, and Facilitated Appropriation only mediates Long-Term Orientation. H18 and $\mathrm{H} 23$ were supported by empirical data. H13-H17, and H19-H22 were not supported by the data.
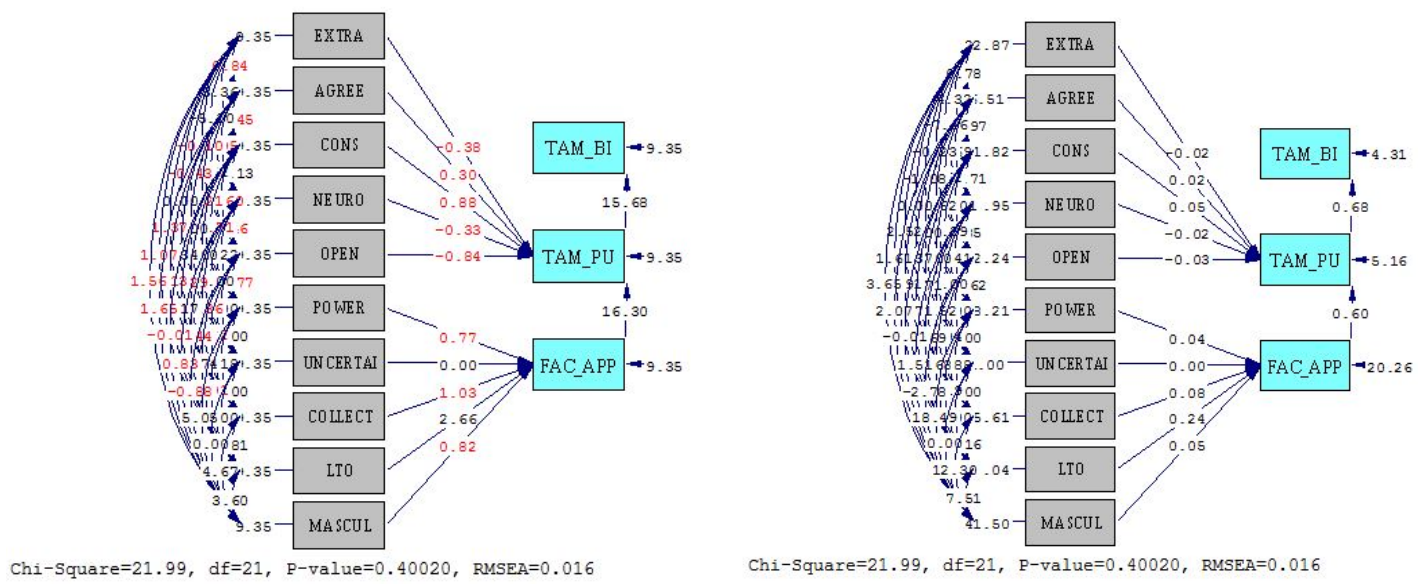

Figure 3. Path analysis results: $t$-value (left) and coefficient estimation (right)

LISREL Estimates (Maximum Likelihood)

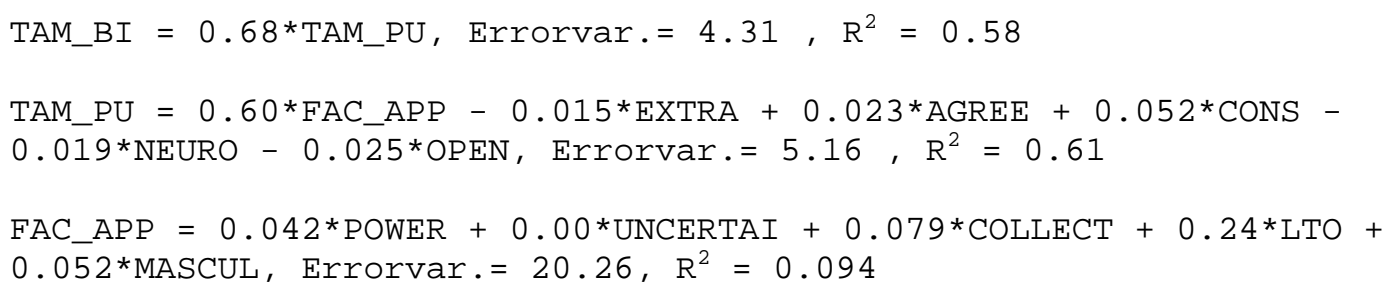

Equation 1. Path analysis result by LISREL: Regression equation 
Notes for Figure 3 and Equation 1. EXTRA = Extraversion; AGREE = Agreeableness; CONS = Conscientiousness; NEURO = Neuroticsm; OPEN = Opennes; POWER $=$ Power Distance; UNCERTAI = Uncertainty Avoidance; COLLECT = Collectivism; LTO = Long-term Orientation; MASCUL = Masculinity; FAC_APP $=$ Facilitated Appropriation; TAM_PU = Perceived Usefulness; TAM_BI = Behavioral Intention; Errorvar. $=$ Error variance; $\mathrm{R}^{2}=$ Coefficient of determination (Effect size).

For additional analysis, the correlation between Age and Behavioral Intention $(r=-0.043, p=0.560$, $p>0.05)$, Perceived Usefulness $(r=0.013, p=0.859, p>0.05)$, and Facilitated Appropriation $(r=-0.050, p$ $=0.494, p>0.05)$ were also computed.

The results were quite interesting, as some of it go against previous studies' finding. We will start discussing our supported hypotheses. It's not surprising that we found, as the model and previous studies suggest that if people find the technology beneficial or provide good use for them, they intend to use it [17]. It's the same for IoT health Device. If people realize it's beneficial for something as important as their health, they will be more likely to use it. Thus, we suggest, in encouraging people to use health device, emphasizing main health benefits should be the top priority.

The only factors we found that would be able to predict user's Perceived Usefulness of device is the Facilitated Appropriation. Giving the fact that if people are facilitated [28] - meaning they are provided with resources and support such as internet access and elaborate information to use the device-combined with their tendency to appropriate, and to relate the device's usage to their personal problem, they will be more likely to perceive the device as useful. The reason is, as mentioned above, appropriating creates additional and more personal value and benefit for themselves, and thus perceiving the device to be more useful than it's designed [27].

However, we must distinguish between general appropriation and facilitated appropriation. Appropriation in nature gives freedom for users to utilize the devices any way they wanted [30], might be as mentioned above, to use sleep tracker to plan schedules and focusing energies on a certain task, or simply to test their body's limit. For example, by understanding how we sleep, how much sleep we need or lack accurately in real-time, users could decide when, how long to sleep, and prepare better for the upcoming task. Similarly, users could keep track of their body changes when to test the effect of certain acts, for example, trying new diets, new supplements, new exercise, or simple to see how long the body can take it or adjust without sugar, fat or even exercising. On the other hand, the concept of Facilitated Appropriation is more guided and better-informed form of appropriating. The options are not limited to user's desires and needs but also combined with shared information, other resources that make appropriating more viable and versatile. Thus, we suggest to increase perceived usefulness towards a device; there's a need to endorse a sense of facilitated appropriation. For example, by emphasizing strong informational and technical support for users to optimize the technology freely according to their own will, and the benefits obtained are only limited by their creativity. For further research, it is recommended to study the impact of facilitation and appropriation processes separately, not as a whole construct, to gain a better understanding of facilitation and appropriation themselves. For example, by examining the mediating effect of facilitation between appropriation and its predictors.

What influences the Facilitated Appropriation? We found that individual's degree of Long-term Orientation (LTO) predicts their tendency to engage in facilitated appropriation. LTO is a cultural orientation that values prospects, people gather information and invest efforts to reach future, distant goal [41]. To reach their future goal, plans and guidance are needed to ensure the goal's attainment. This is where IoT device plays the important part: to help people reach their long-term goal step by step, to plan, to strategize and bring an alternative for people to invest energy on for the sake of the future goal. But why Facilitated Appropriation? Here, we argue that appropriation of the device is positive in nature, as using the device differently from designed purposes to meet own needs and goal will help an individual attain the goal faster and better. For the sake of future fruition, individuals with long-term orientation are very thrifty and perseverance [41]. As mentioned above, health goals are considered long-term goals that need time and efforts to be invested periodically. With enough resources to do so and appropriate, individuals will be more ensured to achieve their long-term health goals.

Next, we will discuss the insight we gained from finding out that personality traits do not predict user's perceived usefulness. Perceived usefulness is indeed dependent on the alignment of information was given, and the goal individual has; however, personality plays an insignificant role. Therefore, for encouraging the use IoT health devices based on its usefulness personality traits could be ignored as relevant factor; however, perceived benefits from the original features or appropriated are the important ones. We came up with a few explanation for these results.

First, personality traits like Openness, Extraversion, Conscientiousness and Agreeableness indicate dispositional characteristics possessed by everyone in responding towards the object [23], for example, in this case, IoT health devices. However, perceived usefulness - a feeling or a tendency to perceive something as 
useful-is a form of belief towards the technology [17], which means it is constructed and formed by individuals, often through experience that forms a certain perspective (useful or not) towards an object that will eventually affect individual's attitude and behavior [47].

As something that is constructed through experience, belief is created through a learning process by actively engaging and the exposure of the device [48]. In an attempt to explore and understand the device, users could learn the device's compatibility-the degree to which a technology is perceived as being consistent with existing values, needs, and experiences of the users (Moore and Benbassat, as cited in [49]), the easiness and practicality in using it, and the information and instructions provided and shared [49]. By the efforts of learning, finding out (experiencing), and mastering the IoT health device in accordance with users' goals, they could construct and determine whether they perceive the device as useful. It makes more sense to think about perceived usefulness as something that is learned and not determined by individual's dispositions or personality traits, at least in the context of this study.

This finding shades a light that in endorsing technological use, deterministic reliance on personality is irrelevant, and concern on how user perceives the benefits of the devices align with their need is the most important one. Regardless of any personality dominant traits, anyone with any dispositions has an equal chance to learn and to perceive the IoT health device as useful and eventually use it.

Some cultural dimensions do not influence appropriation. We previously thought that people in a culture that are likely to avoid ambiguous situation will be less likely to appropriate. However, as the result contradicted with our hypothesis, we propose that there are two paradoxical natures exists in the relationships between the appropriation and Uncertainty Avoidance.

Ambiguity can somehow support the appropriation. Ambiguity can be found all over the place, as most things we encounter in life is inherently ambiguous-meaning they can be interpreted in many ways [50]. The same goes for the situation where we encounter a device like IoT health trackers. How the features work to help individuals in achieving their unique, context-specific situation health goals were not stated in the guiding manuals, as problems faced and goal set vary across individuals. Thus, how the device will be used by individuals are 'open to interpretation', making the ambiguity of the device indeed the 'opportunity' for further appropriation. However, on the other hand, these devices were designed to interact and collaborate with the users that promote certain communicative acts, such as asking question, problem alarming and provide answers/alternatives. Moreover, in many health trackers, there is a function for the device to adapt to the user's health behavior pattern, making the process structure of the device to guide the content, pattern or timing of the communication and interaction better (compare also with [51]). This interaction process provides a clearance and better understanding for the user to understand and interact better with the device. Ambiguity/uncertainty in some phases of IoT use that goes along with the clearance in other phases provided by the device's function might make the absence of predictive correlation between uncertainty avoidance and perceived usefulness.

The result also indicates that Collectivism, as we argued could be endorsing inter-dependent sharing of information and activities, did not have an influence on appropriation. IoT health device such as health tracker is a personal tool for personal benefits gain, and except for its additional social sharing features, it does not involve other people when it comes to using the device as there might be more important and collectively relevant information other than using a personal health device to share.

As for the lack of prediction ability for Power Distance, we reasoned that as health trackers are goaloriented, utilitarian technology for achieving personal goals [52], thus whether the gap of subordinates and authority is small or large, power over others in using this personal technology is irrelevant. Taken together of these explanations, although it can be social, using IoT devices such as health trackers are very personal practices as its benefits are inseparable with our daily life activities.

We propose the reason that espoused Masculinity/Femininity values in individuals did not predict their tendency to engage in facilitated appropriation lies on their main characteristics. As mentioned in the previous section, masculine values emphasize work goals such as earnings, advancement, performance and assertiveness; and individuals with feminine values tend to emphasize personal goals such as a friendly atmosphere, quality of life, and warm personal relationships [31]. We argued that both values might support the motives of appropriating, as feminine value that imposes the quality of life and the personal relationship might find ways to appropriate the device to enhance their life quality. For example, the use of the device is not necessary limited to their own health, as they might use the device as a source to help (e.g. giving health advice) other people, based on what they learned from it. This explanation is also supported by Gefen and Straub [53] which stated that individuals with low masculinity tend to sympathize and care about the weaker members, and focus on social leveling.

For additional analysis, we tried to correlate age factor with Behavioral Intention, Perceived Usefulness, and Facilitated Appropriation. As the age span of the sample in this study was quite large and many studies have considered age to play an important part in technology acceptance, we suppose there are 
some differences in their acceptance towards the technology, namely the intention to use the device, perceived usefulness, and their tendency to engage in facilitated appropriation. However, we found no correlation between age and the three variables. We suggest here might be other variables playing roles in between the age and technology acceptance dimensions that were not measured in this study, such as cognitive abilities [54] and the meaning of age for the individuals [55]. Modern IoT (e.g. [56]) and technology acceptance (e.g. [57]) further study is recommended to consider our present research results and discussions.

\section{CONCLUSION}

We conclude that in this study, user's intention to use IoT health device depends on their perceived usefulness of the device. This means the more useful they see the device, the more likely they will use it. The only things we found to influence this belief or perception is their tendency to appropriate the technology with facilitation. Individual with Long-Term Orientation cultural orientation would be more likely to engage in the facilitated appropriation. However, personality traits is irrelevant and should be disregarded in endorsing the use of IoT health device.

This study can be seen as a suggestion for parties that seeks to encourage the use of IoT health devices, such as medical institutions, companies, and medical practitioners. The main suggestion here is emphasizing on benefits is extremely important to endorse use or acceptance. The benefit should also align with the device's guideline and operation to maximize the potential appropriation for the users not only for small scale short term goals but specifically and strongly on longer term goal. If the device should contain peer-sharing features, it should function mainly to share benefits such as health goals attainment, progress and maybe new ways to appropriate.

\section{ACKNOWLEDGMENT}

We would like to thank Vivi Angelina, Frengky Johanes, Ezra Redo Jawentinus, Virgon Hartoyo, and Marcellino Yohanes, who assisted us in the initial stage of this study.

\section{REFERENCES}

[1] S. M. R. Islam, et al., "The Internet of Things for Health Care: A Comprehensive Survey," IEEE Access, vol. 3, pp. 678-708, 2015.

[2] A. Whitmore, et al., "The Internet of Things - A survey of topics and trends," Information Systems Frontiers, vol/issue: 17(2), pp. 261-274, 2014.

[3] V. K. Abinaya and Swathika, "Ontology Based Public Healthcare System in Internet of Things (IoT)," Procedia Computer Science, vol. 50, pp. 99-102, 2015.

[4] C. E. Turcu and C. O. Turcu, "Internet of Things as Key Enabler for Sustainable Healthcare Delivery," Procedia Social and Behavioral Sciences, vol. 73, pp. 251-256, 2013

[5] B. L. Iantovics, "Agent-based Medical Diagnosis Systems," Computing and Informatics, vol/issue: 27(4), pp. 593$625,2008$.

[6] M. S. Hagger, "Self-regulation: An Important Construct in Health Psychology Research and Practice," Health Psychology Review, vol/issue: 4(2), pp. 57-65, 2010.

[7] T. Mann, et al., "Self-Regulation of Health Behavior: Social Psychological Approaches to Goal Setting and Goal Striving," Health Psychology, vol/issue: 32(5), pp. 487-498, 2013.

[8] N. Bui and M. Zorzi, "Health Care Applications," Proceedings of the 4th International Symposium on Applied Sciences in Biomedical and Communication Technologies - ISABEL '11, 2011.

[9] G. Dobrijević and J. Đ. Boljanović, "Negotiation Goes High Tech: Can You Negotiate with A Machine?," Proceedings of the 1st International Scientific Conference - SINTEZA, 2014.

[10] F. D. Davis, "A Technology Acceptance Model for Empirically Testing New End-User Information Systems: Theory and Results," Ph.D. Dissertation, Massachusetts Institute of Technology, Sloan School of Management, 1986.

[11] M. Kim, et al., "Private Club GMs'/COOs' Perceptions in Adopting Social Media: Applying the Technology Acceptance Model," Journal of Tourism and Hospitality Management, vol/issue: 4(1), pp. 37-48, 2016.

[12] E. Basak, et al., "Examining the Factors Affecting PDA Acceptance among Physicians: An Extended Technology Acceptance Model," Journal of Healthcare Engineering, vol/issue: 6(3), pp. 399-418, 2015.

[13] K. Behrenbruch, et al., "Understanding Diversity - The Impact of Personality on Technology Acceptance," Human-Computer Interaction - INTERACT 2013, pp. 306-313, 2013.

[14] O. Nov and C. Ye, "Users' Personality and Perceived Ease of Use of Digital Libraries: The Case for Resistance to Change," Journal of the American Society for Information Science and Technology, vol/issue: 59(5), pp. 845-851, 2008.

[15] A. M. Anetta, et al., "Using the Theory of Technology Acceptance Model to Explain Teenagers' Adoption of Smartphones in Transylvania," Studia UBB Negotia, vol. 57, pp. 3-19, 2012. 
[16] I. Im, et al., “An International Comparison of Technology Adoption,” Information \& Management, vol/issue: 48(1), pp. 1-8, 2011.

[17] G. B. Svendsen, et al., "Personality and Technology Acceptance: The Influence of Personality Factors on the Core Constructs of The Technology Acceptance Model," Behaviour \& Information Technology, vol/issue: 32(4), pp. 323-334, 2013.

[18] R. Walczuch, et al., "The effect of service employees' technology readiness on technology acceptance," Information \& Management, vol/issue: 44(2), pp. 206-215, 2007.

[19] A. C. Punnoose, "Determinants of Intention to Use eLearning Based on The Technology Acceptance Model," Journal of Information Technology Education: Research, vol/issue: 11(1), pp. 301-337, 2016.

[20] S. Devaraj, et al., "Research Note - How Does Personality Matter? Relating the Five-Factor Model to Technology Acceptance and Use," Information Systems Research, vol/issue: 19(1), pp. 93-105, 2008.

[21] T. Teo, et al., "A Cross-Cultural Examination of the Intention to Use Technology between Singaporean and Malaysian Pre-Service Teachers: An Application of The Technology Acceptance Model (TAM)," Educational Technology \& Society, vol/issue: 11(4), pp. 265-280, 2008.

[22] P. A. Rosen and D. H. Kluemper, "The Impact of The Big Five Personality Traits on The Acceptance of Social Networking Website," in Americas Conference on Information Systems, Toronto, ON, Canada, pp. 1-10, 2008.

[23] R. Kaynak and S. Ekşi, "Effects of Personality, Environmental and Health Consciousness on Understanding the Anti-consumptional Attitudes," Procedia - Social and Behavioral Sciences, vol. 114, pp. 771-776, 2014.

[24] M. Khan, et al., "Exploring the Influence of Big Five Personality Traits towards Computer Based Learning (CBL) Adoption," Journal of Information Systems Research and Innovation, vol. 8, pp. 1-8, 2014.

[25] V. Özbek, et al., "The Impact of Personality on Technology Acceptance: A Study on Smart Phone Users," Procedia - Social and Behavioral Sciences, vol. 150, pp. 541-551, 2014.

[26] P. T. Costa Jr. and R. R. McCrae, "Domains and Facets: Hierarchical Personality Assessment Using the Revised NEO Personality Inventory," Journal of Personality Assessment, vol/issue: 64(1), pp. 21-50, 1995.

[27] A. Dix, "Designing for Appropriation," in BCS-HCI '07, Swinton, UK, pp. 27-30, 2007.

[28] Y. M. Lin, "Understanding Students' Technology Appropriation and Learning Perceptions in Online Learning Environments," Ph.D. Dissertation, University of Missouri, Columbia, 2005.

[29] R. P. Bostrom, et al., "Group Facilitation and Group Support Systems," in Group Support Systems: New Perspectives, 1st ed., L. Jessup and J. Valacich, Ed. New York: Van Nostrand Reinhold, pp. 146-168, 1993.

[30] A. Salovaara and S. Tamminen, "Acceptance or Appropriation? A Design-oriented Critique of Technology Acceptance Models," Future Interaction Design II, pp. 157-173, 2008.

[31] M. Srite and E. Karahanna, "The Role of Espoused National Cultural Values in Technology Acceptance," Management Information Systems Quarterly, vol/issue: 30(3), pp. 679-704, 2006.

[32] L. C. Contiua, et al., "Hofstede's Cultural Dimensions and Student's Ability to Develop an Entrepreneurial Spirit," Procedia - Social and Behavioral Sciences, vol. 46, pp. 5553-5557, 2012.

[33] H. Sriwindono and S. Yahya, "Toward Modeling the Effects of Cultural Dimension on ICT Acceptance in Indonesia," Procedia - Social and Behavioral Sciences, vol. 65, pp. 833-838, 2012.

[34] L. Sagiv and S. H. Schwartz, "Cultural Values in Organisations: Insights for Europe," European Journal of International Management, vol/issue: 1(3), pp. 176, 2007.

[35] M. L. Jones, "Hofstede - Culturally questionable?," in Oxford Business \& Economics Conference, Oxford, UK, pp. 1-8, 2007.

[36] A. Ly, “A Critical Discussion of Hofstede's Concept of Power Distance," SYNAPS - A Journal of Professional Communication, vol. 28, pp. 51-66, 2013.

[37] S. Y. Hsu, et al., "Critical Tests of Multiple Theories of Cultures' Consequences: Comparing The Usefulness of Models by Hofstede, Inglehart and Baker, Schwartz, Steenkamp, as well as GDP and Distance for Explaining Overseas Tourism Behavior," Journal of Travel Research, vol/issue: 52(6), pp. 679-704, 2013.

[38] G. J. Hofstede, "Theory in Social Simulation: Status-Power Theory, National Culture and Emergence of The Glass Ceiling," in Social Coordination: Principles, Artefacts, and Theories, AISB, Exeter, pp. 21-28, 2013.

[39] Y. Altuncu, et al., "Preliminary Study for the Development of Uncertainty Avoidance Instrument in Turkey," Journal of Business, Economics \& Finance, vol/issue: 1(4), pp. 34-48, 2012.

[40] M. C. Gul, "Long-term Orientation, Perceived Consumer Effectiveness, and Environmentally Conscious Consumer Behavior: The Case of Turkey," International Journal of Marketing Studies, vol/issue: 5(5), 2013.

[41] K. H. Brigham, et al., "Researching Long-Term Orientation: A Validation Study and Recommendations for Future Research," Family Business Review, vol/issue: 27(1), pp. 72-88, 2013.

[42] L. Gao and X. Bai, "A Unified Perspective on The Factors Influencing Consumer Acceptance of Internet of Things Technology," Asia Pacific Journal of Marketing and Logistics, vol/issue: 26(2), pp. 211-231, 2014.

[43] O. P. John, "The "Big Five" Factor Taxonomy: Dimensions of Personality in the Natural Language and in Questionnaires," in Handbook of Personality: Theory and Research, 1st ed., L. Pervin, Ed. New York: Guilford Press, pp. 66-100, 1990.

[44] P. Sharma, "Measuring Personal Cultural Orientations: Scale Development and Validation," Journal of the Academy of Marketing Science, vol/issue: 38(6), pp. 787-806, 2009.

[45] B. Yoo, et al., "Measuring Hofstede's Five Dimensions of Cultural Values at the Individual Level: Development and Validation of CVSCALE," Journal of International Consumer Marketing, vol/issue: 23(3-4), pp. 193-210, 2011.

[46] Y. Yung, "Structural Equation Modeling and Path Analysis Using PROC TCALIS in SAS® 9.2," in SAS Global 
Forum: Statistics and Data Analysis, pp. 1-19, 2008.

[47] M. Perepiczka, et al., "Relationship between Graduate Students' Statistics Self-Efficacy, Statistics Anxiety, Attitude toward Statistics, and Social Support," The Professional Counselor: Research and Practice, vol/issue: 1(2), pp. 99-108, 2011.

[48] C. Kynigos, "Constructionism: Theory of Learning or Theory of Design?," Selected Regular Lectures from the 12th International Congress on Mathematical Education, pp. 417-438, 2015.

[49] R. Cheung and D. Vogel, "Predicting User Acceptance of Collaborative Technologies: An Extension of The Technology Acceptance Model for E-learning," Computers \& Education, vol. 63, pp. 160-175, 2013.

[50] W. W. Gaver, et al., "Ambiguity as A Resource for Design," Proceedings of the conference on Human Factors in Computing Systems - CHI '03, 2003.

[51] M. Overdijk and W. van Diggelen, "Innovative Approaches for Learning and Knowledge Sharing," in EC-TEL 2006, pp. 89-96, 2006.

[52] H. M. Colvin, "The Acceptance of Domestic Technology: TAM as Applied to a Proposed Classification Scheme," Ph.D. Dissertation, Iowa State University, 2008.

[53] D. Gefen and D. W. Straub, "Gender Differences in the Perception and Use of E-Mail: An Extension to the Technology Acceptance Model," Management Information Systems Quarterly, vol/issue: 21(4), pp. 389-400, 1997.

[54] K. Chen and A. H. S. Chan, "A Review of Technology Acceptance by Older Adults," Gerontechnology, vol/issue: 10(1), 2011.

[55] S. J. Hong, et al., "How Old are You, Really?: Cognitive vs. Chronological Age in Technology Acceptance Decisions," in SIGHCI, 2008.

[56] S. D. Kim and S. E. Lee, "Little Core Based System on Chip Platform for Internet of Thing," International Journal of Electrical and Computer Engineering, vol/issue: 5(4), pp. 695-700, 2015.

[57] J. Abraham and M. Trimutiasari, "Sociopsychotechnological Predictors of Individual's Social Loafing in Virtual Team," International Journal of Electrical and Computer Engineering, vol/issue: 5(6), pp. 1500-1510, 2015.

\section{BIOGRAPHIES OF AUTHORS}

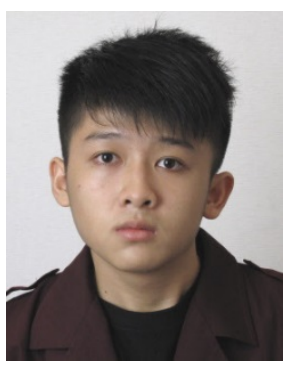

Tommy Prayoga is an alumnus of Psychology Department, Bina Nusantara University; Laboratorium Assistant in teaching practicum class specialized in Test Administration, Observation and Interview Methods; Campus organization trainer specialized in interviewing and recruiting candidates; Senior coach for student psychology competitions in the Department.

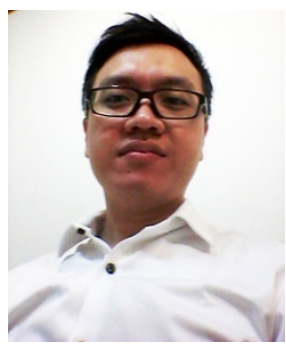

Juneman Abraham is the Faculty Member-Subject Content Coordinator (FM-SCC) on Community Psychology Studies at Bina Nusantara University (BINUS.ac.id); Secretary-General of Asian Psychological Association (APsyA.org); President of Association of Behavioural Researchers on Asians-Indonesia Chapter (ABRAIndonesia.org); Head of Organizational Compartment at Indonesian Psychological Association (HIMPSI.or.id); Certified Webmaster Professional (CWP) of the WOW Academy. Personal page: http://about.me/juneman 